\title{
Uncertainty handling in estimative intelligence - challenges and requirements from both analyst and consumer perspectives
}

\author{
Bjorn G. Isaksen ${ }^{\mathrm{a}, \mathrm{b}}$ and Ken R. McNaught ${ }^{\mathrm{a}}$ \\ ${ }^{a}$ Operational \& Decision Analysis Group, Centre for Simulation \& Analytics, Cranfield University, \\ Defence Academy of the UK, Shrivenham, Swindon SN6 8LA, UK.
}

${ }^{b}$ The Norwegian Defence University College, Akershus Fortress, Building 10, Oslo, Norway.

\begin{abstract}
Important assessments of events and activities relating to military, terrorist and hybrid adversaries, and the intentions of foreign governments, are made every day, usually involving subjective or 'estimative' probabilities and an associated level of confidence. The way in which these uncertainties are assessed and communicated can potentially have enormous impact and consequences. Challenges are reinforced by increasingly complex intelligence problems for which the contemporary analytic paradigm is not tailored to cope. It is important to better understand how defence intelligence analysts and consumers handle uncertainty in their assessment and decision support activities, and what challenges and requirements they face in doing so. This is mainly achieved by the use of semi-structured interviews with a sample of very senior consumers of military intelligence (mostly Flag Officers of the Norwegian Armed Forces) and focus group interviews with groups of Norwegian intelligence analysts. In general, respondents found it difficult or challenging to conceptualize uncertainty analytically. This has implications for the communication of uncertainty and its use in decision-making within the current framework. Secondly, respondents were receptive to suggested potential improvements to the existing framework. One such suggestion involved a differentiated framework, offering different levels of uncertainty resolution in different situations, although none of the respondents had any experience of such a framework for assessing or communicating uncertainty. We conclude with some recommendations to improve the process of uncertainty and risk communication in this important and consequential application area. Having particular implications for policy, we recommend that analysts follow a differentiated approach in handling different situations and problems comprising uncertainty, rather than pursuing a standard solution as is current practice.
\end{abstract}

Keywords: uncertainty; intelligence; military; communication; framework

\section{Introduction}

Different application domains have different approaches for conceptualizing and handling uncertainty in support of decision-making. In this paper, we focus on how defence intelligence analysts and consumers of intelligence (decision-makers) handle uncertainty in their assessments and decision support activities, and discuss the nature of the different challenges and requirements faced by them in doing so. Of particular interest is the degree to which current analytic and tradecraft standards reflect a feasible and acceptable approach to uncertainty handling in a complex operating environment, and how compatible these 
standards are with existing frameworks, from both analyst and consumer perspectives. This is a sparsely populated topic in the intelligence literature.

There is an apparent inconsistency and conflation of terminology related to the term "Estimative Intelligence" in academia as well as in extant doctrines and publications. One of several pathologies within Estimative Intelligence is related to a missing consistent key terminology underpinning existing frameworks for uncertainty and uncertainty handling. Hence, the word "estimate" is often interchanged or conflated with other terminology with different connotations (e.g. "predictions" and "forecasts") signifying the nature of these kinds of intelligence products which is mainly related to assessments of events and activities relating to military, terrorist and hybrid adversaries, and the intentions of foreign governments and other actors. The importance of having a consistent "tribal language" or terminology underpinning this area of uncertainty handling is as important as in other disciplines, e.g. medicine, political science, meteorology and geology. This is also advocated by Lynham ( 2002) as being an essential element in theory building for applied disciplines.

Our objective in this study was to better understand how intelligence analysts and the decision makers, or consumers of that intelligence, handle uncertainty within Estimative Intelligence and what challenges they face in doing so. This was addressed primarily via a series of semi-structured interviews. Expert interviews were conducted with Flag Officers of the Norwegian Armed Forces to obtain the perspectives of intelligence consumers. Focus group interviews with groups of Norwegian intelligence analysts were conducted to obtain the perspectives of analysts.

\section{Background}

\section{Current theory and practice}

Sherman Kent's renowned paper, "Words of Estimative Probability" (Kent 1964), set the agenda for the next 50 years of discourse concerning how to address and handle uncertainty, both analytically and as a means of communicating intelligence assessments to stakeholders and decision-makers. Much of the discussion since then has been about how to establish and communicate uncertainty, e.g. representing likelihood by using verbal descriptors alone or, to reduce ambiguity, using subjective probabilities alone, or, as is currently the case, to use verbal descriptors alongside a subjective probability scale where each descriptor is associated with a range of probabilities (UK MOD 2011; NATO 2016). While these options relate to likelihood information, there is also the need to represent the confidence associated with an assessment. Currently, this is achieved with a verbal scale but in practice, there is some confusion regarding the distinction between likelihood and confidence (Friedman \& Zeckhauser, 2014, 14).

Today, there is still no epistemologically justified theoretical foundation underpinning analytical practices for uncertainty handling in Estimative Intelligence (Vrist Rønn and Høffding 2013). Besides different understandings of the constructs and lack of standardization, there is no framework related to elicitation of subjective probabilities, aggregation of uncertainty including missing and ambiguous information, how to implement likelihood (verbally/ numerically) consistently, or how to derive a consistent measure of 
confidence in assessments.

The current framework for Estimative Intelligence has remained to an extent unchanged over the last four decades. It is mainly approached by a generic taxonomy of five distinct and intertwined analytic paths in the processing of intelligence assessments.

These paths encompass "collation, evaluation, analysis, integration and interpretation" (NATO 2016, 3-10; UK MOD 2011, 3-3), which in turn comprise aggregated uncertainty in finished intelligence assessments.

The core of estimative intelligence involves subjective or 'estimative' probabilities, an associated level of confidence and a source evaluation. The first of these three pillars comprises a source evaluation by using an alphanumeric coding to assess the reliability of the collection capabilities and information sources, and the credibility of the information and data received from these sources (UK MOD 2011; NATO 2016). This form of source evaluation encompasses a wide span of collection capabilities, ranging from human intelligence to signals intelligence.

The second pillar involves the derivation of subjective or 'estimative' probabilities. This is done judgementally and with no established methods or protocols for tailored elicitation of probabilities. Representation of uncertainty in estimative narratives is normally done numerically, or with a verbal description. Often, due to various preferences in terms of preferring numerical versus verbal approaches to uncertainty, a so-called yardstick approach is established. This yardstick uses a rank scale consisting of intervals of numerical probabilities with corresponding words of estimative probabilities (NATO 2016; ODNI 2015; Peterson 2008). The framework is not standardized, and there is a missing consistency in terms of likelihood scales, the meaning of the various verbal probability expressions, and how confidence is assessed and communicated (Jousselme 2016). However, there are several good examples of empirical approaches intended to derive a consistent yardstick. A large project conducted by Canadian Intelligence as well as years of work within climate research clearly identify the challenges by utilizing such a method for communication of uncertainty (Mandel, Barnes, and Richards 2014; Ho et al. 2015; Dhami 2017).

In order to support the measure of uncertainty established and communicated by a subjective probability, it is normally mandatory to provide an associated level of confidence. This is the third pillar. Besides taking account of the quality of underlying intelligence sources, analytic confidence is also intended to account for the analytic methods used and other relevant factors deemed necessary to support analytical reasoning (ODNI 2015; Peterson 2008).

The only resembling facet to uncertainty and uncertainty handling which can be openly accessed within academia, in professional doctrines/ publications and in intelligence directives is the current Analytic Standards, issued by the U.S. Director of National Intelligence (ODNI 2015). These standards and analytic ideals explicitly touch upon many relevant aspects of uncertainty handling. This in turn may be viewed as a good basis, preconditioned on making the standards concrete enough for a realistic applied context. However, the extent to which these standards can be implemented and applied in practice is still an ongoing discourse in academia (Marchio 2014; Gentry 2015). 


\section{Problems with current approach}

Uncertainty handling in estimative intelligence has a number of characteristics, some of which are shared with some other domains, and others which are more distinctive.

One characteristic shared with other domains such as medicine, climate change and disaster risk management is the potential gravity of the consequences of having poor, missing, inconsistent or ambiguous analytic premises as the basis for reasoning under uncertainty.

Another characteristic shared with emergency workers and some law enforcement investigators is the way in which intelligence analysts often must work, in a pressurised, time-constrained environment with insufficient time and resources to elicit and acquire a desired level of knowledge and understanding to support decision-makers. Analysts often must elicit, integrate and interpret incomplete and contradictory information, some of which, in the cases of intelligence and law enforcement, may arise from an adversary's use of deception. These features of intelligence analysis impact the degree of objectivity in analytic judgements, making them more susceptible to undesired cognitive biases (Goodwin and Wright 2004; Heuer 1999; Tversky and Kahneman 1974).

An important dimension in this respect is the understanding, expectations and requirements from intelligence consumers, which vary as a function of the level and type of decision-maker. Hence, decision-makers who are military commanders in an operational setting have different and possibly much more time-constrained intelligence requirements than policy-makers in bureaucratic positions. However, in general, intelligence consumers ought to have a good understanding of how uncertainty is handled and eventually communicated when receiving estimative assessments based upon their own communicated focused intelligence requirements. Otherwise they might assume that the intelligence they have received warrants more, or perhaps less, credibility than is actually the case.

There is much agreement within academia and in military doctrine and publications regarding the nature of security threats, driving forces and how the operating environment has to be viewed and analysed differently relative to the Cold War paradigm of analysis. For example, in NATO, UK and U.S. doctrines and publications, we find similar narratives in terms of the operating environment and the importance of handling analytic uncertainty in intelligence assessments and in operational planning (NATO 2010; SHAPE 2013; Ministry of Defence UK 2014; US Joint Chiefs of Staff 2013). Moreover, the characteristics of problems and challenges facing intelligence communities (ICs), military planners and decision-makers are often reoccurring themes. These narratives agree that the character of conflict has changed to a more complex operating environment with fuzzier operational boundaries, increased uncertainty and non-linear effects. However, besides acknowledging the importance of handling uncertainty consistently and analytically, the resolution is remarkably low concerning how uncertainty is conceptualized in intelligence assessments and operational planning. The latter includes operational assessments with inherent risk assessments.

Due to this perceived shift towards greater complexity in the operating environment, a natural question is whether the current analytic framework for uncertainty handling is sufficient and hence provides the necessary fidelity and analytic rigour to meet analytic standards and decision-maker or intelligence consumers' requirements for decision-making. Many analytic problems facing intelligence communities today comprise what Conklin 
$(2005,5)$ and Moore $(2011,18)$ describe as "Wicked Problems". These unbounded problems are often characterized by non-linearity, adaptivity, social complexity, dynamic actors and feedback mechanisms impacting how problems develop. Operational experience and lessons identified from counterinsurgency settings often serve as a basis for describing such complex operating environments (Connable 2012). For example, the effects of military strikes on insurgency recruitment can vary widely in different situations.

These problems create significant analytical challenges in intelligence analysis, and the current analytic paradigm, advocated by Lahneman $(2010,209)$ as "solving puzzles", is often criticised for not being able to cope with such problems (Connable 2012; Charters, Farson, and Hastedt 2004).

There are few normative or prescriptive guidelines in terms of how to handle such complex and unbounded problems when looking through an analytic lens. This is mainly due to the generalization and hence low fidelity in extant doctrines and publications. The path of least resistance in approaching analytic complexity with its inherent uncertainties is therefore to embark on reductionism, which means a simplistic analytic approach to a complex problem. However, this stands in contrast to the stated level of ambition encompassing a "holistic" approach to analysis along the entire continuum of intelligence problems in order to cover the inherent complexity comprising the operating environment (NATO/ ACT 2011; SHAPE 2013). Despite the ambition, however, there is little concrete guidance as to how this should be achieved.

\section{Research objective and questions}

With the current framework for uncertainty handling, and the dominant set of analytic standards as a basis, the main research objective for this paper was to better understand how intelligence analysts and the decision makers, or consumers of that intelligence, handle uncertainty within Estimative Intelligence. A related objective was to gauge respondents' attitudes to a tailored or differentiated uncertainty handling framework for defence intelligence communities. Such an approach, which we are currently developing, would allow different levels of uncertainty resolution to be pursued in different situations and for the continuum of intelligence problems.

The research questions addressed are:

(1) What is the degree of consistency and analytical quality in the handling of uncertainty within Estimative Intelligence?

(2) What are the main challenges, problems, and requirements related to uncertainty handling within Estimative Intelligence, from both analyst and consumer perspectives?

\section{Method}

\section{Data collection approach}

Semi-structured interviews with intelligence consumers and focus-groups with intelligence analysts were the chosen data collection methods. Besides the sparsity of literature on the 
research topic, another justification for using qualitative methods was to give respondents the opportunity to provide answers within their own frame of reference, insight and understanding (Aberbach and Rockman 2002, 674).

Interview protocols were constructed with an emphasis on eliciting a set of "attitudes, values, beliefs" (Aberbach and Rockman 2002, 673) and approaches related to how uncertainty in Estimative Intelligence is conceptualized, operationalized and coped with in the context of analysis and decision-making in complex military operating environments. The interview protocol further contained a set of probes and prompts to aid clarification, and to help bridge the processes between transcription, coding and analysis. The interview questions set forth in the interview protocols have a funnelling structure with themes in order to increase the coherence of the responses. The questions encompassed current practices, understanding, challenges and requirements related to uncertainty and uncertainty handling in Estimative Intelligence in the context of complex problems.

Intelligence analysts, given the inherent characteristics of their tradecraft, are likely to prefer to be selective in their answers. An open- ended approach to the elicitation process was therefore judged to encourage their willingness to respond, and consequently to yield better data. The data collection was tailored to capture the different perspectives between decisionmakers (intelligence consumers) and intelligence analysts, and to investigate the degree to which there was agreement between them with respect to key issues. The interviews were conducted in Norwegian, and further transcribed and translated into English prior to analysis, integration and derivation of findings.

\section{Sampling strategy}

A purposive sampling method was chosen in order to address the research questions and to exploit the researcher's knowledge and ability to identify, and recruit specific expert and analyst respondents. The main selection criteria entailed a judgmental assessment of relevant background, operational experience, professional reliability and authority. Moreover, all research participants have been carefully selected for their jobs (Tansey 2007, 770-771). Consequently, a subjective and non-probabilistic sampling approach was taken. This controlled selection process formed the basis for the selected sample of respondents with the aim of establishing a focus on key issues within uncertainty handling in Estimative Intelligence.

\section{The main characteristics and size of the sample}

Semi- structured, expert interviews were conducted with 10 intelligence consumers. The majority of the respondents were flag- level (holding the rank or equivalent rank of a general level officer in the army, i.e. brigadier and upwards) decision- makers/ intelligence consumers. Each had extensive decision-making experience, and a comprehensive track record from international service and operations within the framework of NATO or coalition operations. All respondents were Norwegian officers. All participants were on active duty except for two who had recently retired. Two focus groups (one with four and one with three respondents) with active duty professional intelligence analysts were also conducted. A third focus group interview was cancelled due to specific circumstances. However, the planned focus group interview was replaced by an extensive semi-structured interview with a single 
analyst from the same intelligence community. Given the qualitative nature of this research, we judged the sufficiency of the sample size in terms of achieving a satisfactory level of data saturation, i.e. a point where no significantly new information was forthcoming. Furthermore, there was a very limited pool of available analysts and, particularly, decision-makers to draw from.

All of the interviews were tape-recorded in order to be able to focus on the interview, and to ensure that the questions, probes and prompts in the interview protocols could be addressed as deemed necessary. There were no significant issues related to the respondents not being perceived as frank and willing to share their own experiences, beliefs and requirements during the interviews. The interviews were conducted in a wide range of places, ranging from their personal homes to business and official military establishments, all with an atmosphere underpinning a good basis for interviews.

\section{Ethical issues}

There were no identified ethical issues related to the data collection processes, and these were approved by the university ethics committee. All participants received an information package in order to better comprehend the purpose, objectives and focus of the research project. Written informed consent was obtained from all respondents. As an additional layer of confidence building, a Certificate of Confidentiality was issued by the researcher. Implementing an extra layer of confidence and trust was assessed to influence the degree to which the respondents were willing to participate, and to be frank and willing to share the desired information in such a context. The interview protocols without probes and prompts were sent to the respondents beforehand for preparation purposes.

\section{Analysis of qualitative data}

There are few templated approaches for analyzing qualitative data, and various schools advocate different approaches. Information elicited by conducting interviews has been used to identify beliefs, values and new insights to contribute to the body of uncertainty handling within intelligence. As opposed to a deductive approach, an inductive approach was chosen in order to analyze information, extract key findings and to make inferences.

This approach was justified due to the lack of or missing “...predetermined theory, structure or framework" (Burnard et al. 2008, 429) for uncertainty handling in Estimative Intelligence. In turn the collected data was used to structure and conduct the analysis by using a thematic content analysis. The analysis encompassed derivation of “...themes and categories that emerge from the data" (Burnard et al. 2008, 430), which formed the basis for the coding framework. Two coding frameworks, each with separate themes and a derived coding hierarchy, were used for analysis, interpretation and derivation of key findings. The structure and focused questions in each interview protocol simplified the data reduction and coding processes. The analyst and consumer perspectives were each assigned one coding framework. 


\section{Results and Discussion}

To address the research questions, a set of key topics and themes related to uncertainty handling in Estimative Intelligence was derived. There are three main themes which mirror image the coding structure in the two different interview protocols:

- Theme 01: The nature of the modern operating environment

- Theme 02: Uncertainty handling in Estimative Intelligence- challenges and problems with extant practices.

- Theme 03: Potential improvements to current practices in Estimative Intelligence.

\section{Theme 1: The nature of the modern operating environment}

There was a general acknowledgement and a common perception and understanding among all the respondents in terms of how operations have changed since Cold War times. In this context there was an explicit awareness of increased complexity and uncertainty being inherent characteristics of contemporary and future crises and conflicts. There was little deviation from the respondents' answers in this regard compared to the mainstream academic literature, and relevant extant military doctrines and publications, e.g. as described by Odom (2008) and in NATO (2011). However, an interesting counterpoint was given by one of the focus groups (FG 1) that situations are not necessarily more complex but the more understanding we gain of a problem domain, the more uncertainty and complexity are revealed.

Uncertainty as an analytic term and phenomenon encompasses multiple aspects and approaches (Agusdinata 2008; Weiss 2007; Warren E. Walker, Lempert, and Kwakkel 2013). Still, a paradox exists as key doctrines and publications encompassing intelligence and the planning of operations emphasize the importance of treating multi-faceted problems holistically and of handling analytical uncertainty in a rigorous and robust way. This is in order to provide insight and understanding as a basis for producing estimative assessments and for decision-making (SHAPE 2013; NATO 2016; NATO 2011; US Joint Chiefs of Staff 2013). However, within these domains uncertainty handling in complex problems is not addressed with sufficient fidelity or with sufficient guidelines for practical application.

All of the respondents found that it was challenging to grasp the concept of uncertainty due to its inherent character and perceived vagueness. When the respondents were asked about their understanding of the nature of analytic uncertainty and the degree to which it was operationalized, there were few clear answers, and the respondents acknowledged the challenges of addressing and handling the topic in general. A typical quote from one of the decision-makers (DM 3) was:

\footnotetext{
“...uncertainty is a vague term and concept. There is a clear requirement for a more systematic approach to handle uncertainty”. [DM3]
}

Besides perceiving uncertainty as a vague construct which is difficult to conceptualize, none of the respondents could refer to any concrete examples where analytic uncertainty had been operationalized in terms of classifying uncertainties, weighing uncertainties or 
aggregating uncertainties in order to see their relative influence on the problem being assessed. There was no notable difference in answers between the two groups of respondents, making this a highly convergent finding. This is important for our current work which is building towards the development of a more refined framework for uncertainty handling within the intelligence domain.

A starting point in order to remedy the current status of uncertainty within the intelligence domain could be to conduct systematic literature reviews addressing relevant research and general literature "outside" the intelligence domain, and then do the same for literature addressing uncertainty "inside" the intelligence literature. Good examples for such an approach are reviews conducted for the discipline of Impact Assessment and a report surveying research on the use of verbal uncertainty expressions (Druzdzel 1989; Leung et al. 2015). Also relevant is the work of Barnes (2015).

Further problems of a modern operating environment mentioned by both analysts and decision-makers related to the extent of information which is now available, and the large number of information channels beyond innate intelligence assets, create an information overload. This phenomenon is discussed by Duvenage (2010).

\section{Theme 2: Uncertainty handling in Estimative Intelligence- challenges and problems with extant practices}

The current framework for Estimative Intelligence, intertwined with standardized risk and operational planning processes in NATO (and across member nations separately), has a low fidelity in terms of applied and methodological guidance, except for considerable literature on the general judgmental and cognitive aspects of decision-making under uncertainty.

Heuer's (1999) book, Psychology of Intelligence Analysis, brings forward synthesized research from cognitive psychology on issues related to thinking, judgment and cognitive biases in particular. These aspects in studies of surprise attack and intelligence failures have had a sedimented place in academia and military doctrines/ publications and, as a result, been a dominant belief within the intelligence literature for the last two decades.

However, as Marrin (2004) already commented some years ago, this area of research has to an extent "... been mined for insight" (661).

As a result of focusing on these aspects of thinking and decision- making within the intelligence related literature, the findings from research within psychology became a dominant factor in developing analytical techniques which were intended to be tailored to intelligence analysis (Mandel 2009; National Research Council 2010). This heritage is still strong today and, as a result, the intelligence discipline suffers from a poor theoretical and epistemological justification upon which to evaluate and develop approaches to analytic uncertainty, particularly for complex problems or scenarios.

The respondents were asked about key issues related to extant practices in Estimative Intelligence. The majority of the decision-makers interviewed preferred to receive likelihood information in verbal terms. However, the extent to which they related those to the associated numerical scale was not made clear. There was also a common view from them that the communication of analytic confidence lacked transparency. This is perhaps not surprising 
since it is a function of several elements, including source characteristics, assumptions made and analytic methods employed. One decision-maker noted:

"...the main impression [is] that there is a lack of a disciplined approach to derivation and communication of analytic confidence in assessments". [DM 1]

However, as a partial explanation, the first focus group of analysts noted that:

"[There is an] important balance in terms of how much uncertainty is required and relevant to communicate to a consumer. What a consumer requires in terms of details and information about processes, methods and analytical inference underpinning estimates do vary”. [FG1]

It is important to emphasize, however, that the majority of the respondents were clear on some mitigation strategies or quality assurance mechanism to handle uncertainty in both analysis and decision-making. This was mainly related to critical thinking, discussions with and among peers and analysts, and if time permitted, to gain insight into the analytical foundation underpinning estimative assessments. Techniques such as formal peer review processes, devils advocacy, and red-teaming (US Government 2009; Heuer 2005) all serve more or less the same intention and there is plentiful literature related to these. That intention is to minimize biases, to consider alternative explanations and alternatives, and to avoid logical flaws and analytical pitfalls. While these all have a bearing on analytic uncertainty, their relationship to it is often not made explicit.

An interesting point raised by several decision-makers was the impression that analysts had a tendency to present too much intelligence in the form of facts and sometimes appeared reluctant to make probabilistic predictions. One decision-maker highlighted the importance of "daring to be explicit about uncertainties". While there may be behavioural reasons for this, another explanation could be related to today's proliferation of multiple information channels, mentioned earlier. Analysts may simply feel obliged to communicate much of this factual information in case it proves relevant, or for fear of otherwise being considered uninformed. One decision-maker noted:

“...in complex problems, [there is] a presence of hesitancy to handle uncertainty and to predict versus traditional emphasis on facts/ what is known. Hence, [there is] a general hesitancy to enter the "room of uncertainty "in estimative assessment". [DM 3]

\section{Theme 3: Potential improvements to current practices in Estimative Intelligence}

A set of questions was posed to the respondent groups to assess the potential for some suggested improvements in order to enhance the existing framework for Estimate Intelligence.

It is current practice in military operational planning processes to derive and evaluate a plan based on considering at least two possible courses of action of an adversary: the most likely and the most dangerous course of action (SHAPE 2013; US Joint Chiefs of Staff 2009). An estimative assessment is fused into this planning process, which also has a deliverable in terms of presenting a limited number of courses of action, seen through the prism of an opposing actor or belligerent (US Joint Chiefs of Staff 2009). Risk management and thus uncertainty handling is also an inherent part of these processes in terms of evaluating the criterion of acceptability related to the dimensions of risk-to-force and risk-to-mission 
(NATO 2018; SHAPE 2013). In the context of risk management, estimative assessments are applied in order to consider the likelihood of events occurring and unfolding.

This is an example of how complex problems are subject to uncertainty handling by using a reductionist approach. A complicated or complex problem is reduced to something that can be handled analytically given the specific constraints of time, insight/ understanding and resources. Current methodology is based on extracting a small number of possibilities from an infinite space of possibilities, and then focusing on refining these alternatives until a decision is made. While Lahneman (2010) describes the current analytic paradigm as not having developed along with the changing operating environment for several decades, Connable $(2012,1)$ reinforces this critique by suggesting a failure of intelligence communities to assess complex operating environments. This inflexible and simplistic approach to complex problems in particular is criticised by several authors.

If time and resources allowed, most decision-makers had a general preference for being presented with multiple alternatives/ scenarios, each with a measure of likelihood and associated analytic confidence attached. This was to be able to make their own judgements given the relevant factors and assessments being presented. One decision-maker said:

"... if time and resources permit, there is a moral obligation by a commander in operations to consider multiple explanations/ possibilities in order to make good judgments. Being only presented with a most likely and most dangerous course of action as an analytical "gold nugget" is often not sufficient in an operational planning phase”. [DM 9]

It was emphasised by several decision-makers that this preference was dependent on the time available and also on the degree of risk present. One decision-maker noted:

“In high risk decisions, [there is] a higher demand for insight and understanding”. [DM 4]

A related issue concerns the derivation, handling and purpose of analytic confidence in estimative assessments. All respondents agreed on the importance of knowing whether or not further collection and analysis would yield any difference in terms of reducing analytic uncertainty to the point where a different decision might be taken. Thus, the respondents clearly required an assessment from analysts if any additional resources spent were likely to only yield a marginal difference to the level of uncertainty. This is paramount to know in decision-making processes in order to avoid missing time-limited opportunities, and in the allocation of scarce resources to maximize effects. The focus group respondents (analysts) clearly agreed with the decision-makers in terms of the importance of this issue. This aspect relates to decision sensitivity, and is also addressed by Friedman and Zeckhauser (2014) who suggest using analytic confidence as a way of expressing “...the value of additional information" (89).

Decision sensitivity also directly relates to the risk management framework in terms of allocation of scarce resources to try and mitigate risk that is not mitigatable. It was mentioned that trying to reduce uncertainty or mitigate risk may in certain cases result in a reactive posture, which in turn may influence the required operational freedom. DM 5 notes: 
“...if there is too much focus on risk, trying to mitigate all derived risks, we can end up with a scattering of focus and resources which in turn can be counterproductive in terms of achieving desired effects in an operational context". [DM 5]

Decision sensitivity could also be linked to a more user-driven approach to uncertainty handling (Gabbert et al. 2010). This may imply that decision-makers explicitly communicate their requirements to intelligence analysts in terms of their need for uncertainty information. Another relevant link to extant research concerns how decision-makers form coping strategies as a function of being presented with uncertainty in assessments (Lipshitz and Strauss 1997). This could provide a useful basis for further investigation.

We were interested in gauging the participants' views regarding a more differentiated approach to uncertainty handling. The majority of the respondents from both interview groups had not been exposed to or used any differentiated framework for uncertainty handling in which analytical requirements and consumer requirements/ expectations had been operationalized as a function of, for example, the problem complexity and scenario specific operational constraints. It was noted by DM2 that:

"Such a framework must be flexible in terms of operational constraints, type of problem, types of intelligence and types of consumers”. [DM 2]

Given the characteristics of the existing framework for uncertainty handling, these findings provide support for the potential usefulness and relevance of developing a tailored framework for uncertainty handling in Estimative Intelligence. Nonetheless, some of the respondents among the analysts were clearly sceptical to embark on another layer of time-consuming analytical processes. Furthermore, some were concerned that this might somehow impact negatively on the analysts themselves:

“...by introducing new methods/tools, the analytic organization may end up in relying too much on the methods/ tools and as a consequence lower the requirements to analysts handling the tools”. [FG 1]

In fact, any such change would almost certainly increase requirements for analyst capability. However, their answers also indicated a positive attitude and willingness to consider such a framework from the majority of the analyst respondents.

Several of the decision-makers had a general preference for being presented with simplistic models and graphical representations. However, there were no examples provided of any particular graphical representations of uncertainty. Only one decision-maker explicitly called for the use of more sophisticated models by analysts:

"Harder methods or models may be important for intelligence communities to use in order to better embrace the dynamic, uncertain and complex operating environment". [DM 3]

This, together with the general preference for verbal representation of uncertainty by most of the decision-makers, suggests that careful thought will be required concerning how the outputs from any differentiated approach are communicated. Looking and learning from other disciplines should be an important part of any such framework development. Some frameworks assessed to be relevant can be found in model-based decision-support (Walker et al. 2003), the European Food Safety Authority (EFSA) guidance on uncertainty (Benford et al. 2018) and within the Intergovernmental Panel on Climate Change (IPCC) in context of 
IPCC guidance notes (Mastrandrea et al. 2010).

Even though an improved uncertainty framework may help to provide better insight and understanding of analytic uncertainty, and consequently an enhanced foundation for decisionmaking, it is also important to decide whether or not this is a tool for analysts alone, or if the framework ought to have a combined intention, taking into consideration requirements set forth by a variety of intelligence consumers requiring estimative assessments with different levels of fidelity, and serving various purposes.

\section{Conclusions, Policy Implications and Recommendations}

The current framework for uncertainty handling in Estimative Intelligence has remained more or less unchanged over the last five decades. Moreover, the framework is simplistically constructed, and not theoretically justified to enable the applied dimension of the tradecraft to evolve naturally as a result of joint efforts between academics and practitioners.

There is considerable revitalized research available and ongoing academic discussion devoted to, for example, whether to use words, numerical probabilities or both to assess and communicate subjective likelihood assessments, and how to best approach the notion of confidence in such assessments. Given the increased requirements for quality, expectations from consumers and the extent to which the analytical standards for uncertainty handling are realistic and can be complied with, relatively little research has been devoted to uncertainty handling from the perspectives of decision-makers and analysts explicitly. Notable exceptions, concerning the communication of uncertainty in particular, are provided by Dhami (2017), Ho et al. (2015) and Mandel and Barnes (2014).

A number of conclusions can be drawn from this study which drew information from serving intelligence analysts and senior military decision-makers with extensive operational experience.

1. Among intelligence analysts and consumers, there is a general acknowledgement and an understanding of how operations have changed in contemporary scenarios and, consequently, an awareness of increased levels of complexity and uncertainty related to Estimative Intelligence. The proliferation of information channels outwith a force's own intelligence function can create additional problems.

2. There is a general challenge for both intelligence analysts and consumers in conceptualizing uncertainty due to its inherent character and perceived vagueness. The lack of transparency related to the meaning, derivation and communication of analytic confidence was a particular concern for decision-makers.

3. Several decision-makers felt that analysts had a tendency to provide a great deal of factual information but were less comfortable in providing predictions. This may be partly due to the proliferation of information mentioned above.

4. There was agreement among analysts and decision-makers that it would be useful to know more about the likely change in uncertainty which further intelligence gathering might bring and any subsequent effect on decision-making.

5. From decision-makers there was support for the provision of multiple assessments rather than just two assessments, based on the most likely and most dangerous actions of an 
adversary. However, they were also clear that this should only be when time permits. This in itself is evidence of support for a differentiated approach.

6. There was little experience of a differentiated approach to uncertainty handling among either analysts or decision-makers. There was interest shown, however, and an understanding of why this might be useful. Some analysts were concerned about the possible impact of such an approach on them.

\section{Policy implications of our findings}

Based upon the findings from the study, together with a comprehensive literature review of uncertainty handling in Estimative Intelligence, the main implications for practice are related to further requirements for the conceptualization and operationalization of uncertainty. These are needed in order to meet changing requirements for analytic uncertainty handling, increased expectations for quality in evidence-based decision-making, and an acknowledgement of the complexity typical of contemporary intelligence problems. They are particularly needed to meet consumer requirements for transparency and understanding of the underpinning uncertainties around estimative assessments. Moreover, better analytic traceability provides a better basis for conducting and communicating analysis and assessments in a collaborative environment, across intelligence communities and other cooperative entities. The rather fixed nature of the extant framework inhibits flexibility and transparency.

In order to overcome these limitations, we believe that a more systematic and differentiated approach to uncertainty handling in the intelligence domain is required. With such an approach, different levels of uncertainty resolution (e.g. regarding the type and location of the uncertainty) could be communicated depending on scenario characteristics and operational constraints, such as available time.

\section{Recommendations}

Further research is needed to improve the level of transparency in the communication of uncertainty between analysts and consumers and to provide clearer guidance on how current analytic standards can be operationalized in practice.

It is also recommended to seek good practice from other domains and disciplines with a view to developing a differentiated uncertainty handling framework which is suitable for defence intelligence communities. Such a tailored framework for uncertainty handling should be viewed as a complement to and evolution of the current framework for Estimative Intelligence, and should take account of the continuum of intelligence problems, domain specific operational constraints and decision-maker requirements.

The current framework used by the IPCC is one good example of a relevant framework to consider (Budescu et al. 2014; Mastrandrea et al. 2010). In addition, conceptual frameworks and models for handling of uncertainty in model-based decision-support (Walker et al. 2003; NATO RTO 2012), and in food safety (Benford et al. 2018; Lofstedt, McLoughlin, and Osman 2017), both comprise a good and relevant basis for framework development of uncertainty handling in Estimative Intelligence. To incorporate how 
Estimative Intelligence is intertwined with operational planning and risk management, it is also recommended to evaluate how Walker, Haasnoot, and Kwakkel (2013) advocate approaches related to Adaptive Policy Making (APM) and Robust Decision Making (RDM). We have already started work on developing such a new framework, and will report on its development in due course.

Besides increasing awareness and transparency in analytical processes, such a framework is also expected to provide a better basis upon which to evaluate and empirically test the large number of Structured Analytical Techniques (SAT) developed for the intelligence domain, with an emphasis on how they handle uncertainty in the context of complex problems.

\section{Limitations of the study}

The inherent nature of qualitative research is in general subject to criticism. As Anderson (2010) emphasizes, rigor is "...difficult to maintain, assess and demonstrate" (2). Two apparent limitations of the study are related to the characteristics of the selected sample and the sample size. A consequence of purposive sampling is susceptibility to selection bias, which in turn can influence the degree of "robust findings and generalizations" (Tansey 2007, 768). However, in this case our samples come from small, relatively homogeneous groups, within which the members have received similar training in the intelligence domain. In addition, many of them will have similar levels and types of operational experience. Perhaps partly because of this, it was noticeable that there was considerable agreement in their responses. This suggests that larger samples are unnecessary as long as the aim is not to generalize to a wider population, such as all NATO officers or all decision-makers (including civilians) receiving this kind of intelligence. Even then, given common operating procedures for handling intelligence within NATO, any differences would most likely be caused by national or cultural differences.

Complementing the sample type by conducting expert interviews with senior civilian policy-makers and intelligence consumers (e.g. politicians) could yield additional insight and new perspectives but access to this group would be more difficult. Lastly, besides enhancing analytical rigour by, for example, a triangulation approach, there are always limitations of time, resources and availability of respondents which in turn constrain all research projects.

\section{Acknowledgements}

We thank both referees and a guest editor for their advice and constructive suggestions which have helped to improve the content and structure of this paper. We also thank the interviewees and focus group participants for giving so freely of their time and opinions based on extensive operational experience. Preliminary findings from this study were first presented at the Cambridge Risk and Uncertainty Conference in June 2017. 


\section{References}

Aberbach, J. D., and B. Rockman. 2002. "Conducting and Coding Elite Interviews." PS: Political Science and Politics 35 (4): 673-76. doi:10.1017.S1049096502001142.

Agusdinata, D. B. 2008. "Exploratory Modeling and Analysis- A Promising Method to Deal with Deep Uncertainty.” PhD thesis, Delft University of Technology. http://www.narcis.nl/publication/RecordID/oai:tudelft.nl:uuid:8a371ab4-3582-4506bbd2-685e127f8bd7.

Anderson, Claire. 2010. "Presenting and Evaluating Qualitative Research: Strengths and Limitations of Qualitative Research.” American Journal of Pharmaceutical Education 74 (8): 1-7. doi:10.5688/aj7408141.

Barnes, Alan. 2015. "Making Intelligence Analysis More Intelligent: Using Numeric Probabilities." Intelligence and National Security 4527 (September): 1-18. doi:10.1080/02684527.2014.994955.

Benford, Diane, Thorhallur Halldorsson, Michael John Jeger, Helle Katrine Knutsen, Simon More, Hanspeter Naegeli, Hubert Noteborn, et al. 2018. "Guidance on Uncertainty Analysis in Scientific Assessments." EFSA Journal 16 (1): 1-39. doi:10.2903/j.efsa.2018.5123.

Budescu, David V., Han-Hui Por, Stephen B. Broomell, and Michael Smithson. 2014. "The Interpretation of IPCC Probabilistic Statements around the World." Nature Climate Change 4 (6): 508-12. doi:10.1038/nclimate2194.

Burnard, P., P. Gill, K. Stewart, E. Treasure, and B. Chadwick. 2008. "Analysing and Presenting Qualitative Data.” British Dental Journal 204 (8): 429-32. doi:10.1038/sj.bdj.2008.292.

Charters, David A., Stuart A. Farson, and Glenn P. Hastedt. 2004. Intelligence Analysis and Assessment. Abingdon, Oxon: Frank Cass Publishers.

Conklin, Jeff. 2005. "Wicked Problems and Social Complexity." Part I, Chapter 1 in Dialogue Mapping: Building Shared Understanding of Wicked Problems, 1st ed., 25. Chichester, West Sussex, England: John Wiley \& Sons Ltd. http://cognexusgroup.com/wp-content/uploads/2013/03/wickedproblems.pdf.

Connable, Ben. 2012. "Military Intelligence Fusion for Complex Operations- A New Paradigm.” RAND Occasional Paper 377. Santa Monica, CA: RAND Corporation. http://www.rand.org/content/dam/rand/pubs/occasional_papers/2012/RAND_OP377.pdf 
Dhami, Mandeep K. 2017. "Towards an Evidence-Based Approach to Communicating Uncertainty in Intelligence Analysis." Intelligence and National Security 33 (2): 257-72. doi:10.1080/02684527.2017.1394252.

Druzdzel, Marek J. 1989. "Verbal Uncertainty Expressions: Literature Review.” Technical Report CMU-EPP-1990-03-02, Department of Engineering and Public Policy. Carnegie Mellon University, Pittsburgh, PA, May 1989.

http://www.pitt.edu/ druzdzel/psfiles/verbal.pdf.

Duvenage, Magdalena Adriana. 2010. "Intelligence Analysis in the Knowledge Age: An Analysis of the Challenges Facing the Practice of Intelligence Analysis." M. Phil. thesis, Stellenbosch University 2010.

https://scholar.sun.ac.za/bitstream/handle/10019.1/3087/duvenage-m-a2010.pdf? sequence $=3$.

Friedman, Jeffrey A., and Richard Zeckhauser. 2014. "Handling and Mishandling Estimative Probability: Likelihood, Confidence, and the Search for Bin Laden." Intelligence and National Security 30 (1): 77-99. doi:10.1080/02684527.2014.885202.

Gabbert, Silke, Martin van Ittersum, Carolien Kroeze, Serge Stalpers, Frank Ewert, and J. A. Alkan Olsson. 2010. 'Uncertainty Analysis in Integrated Assessment: The Users' Perspective." Regional Environmental Change 10 (2): 131-43. doi:10.1007/s10113-0090100-1.

Gentry, John A. 2015. "Has the ODNI Improved U.S. Intelligence Analysis?” International Journal of Intelligence and CounterIntelligence 28 (4): 637-61. doi:10.1080/08850607.2015.1050937.

Goodwin, Paul, and George Wright. 2004. Decision Analysis for Management Judgment. 3rd Edition. Chichester, West Sussex, England: John Wiley \& Sons Ltd.

Heuer, Richards J. 2005. "Limits of Intelligence Analysis.” Orbis 49 (1): 75-94. doi:10.1016/j.orbis.2004.10.007.

Heuer, Richards J. 1999. Psychology of Intelligence Analysis. Lulu.com. http://www.google.no/books?hl=en\&lr=\&id=NWwkQoHlsCcC\&pgis=1.

Ho, Emily H., David V. Budescu, Mandeep K. Dhami, and David R. Mandel. 2015. "Improving the Communication of Uncertainty in Climate Science and Intelligence Analysis." Behavioral Science \& Policy 1 (2): 43-55. doi:10.1353/bsp.2015.0015.

Jousselme, Anne-Laure. 2016. "Presentation of Uncertainty Representations." NATO SAS114 Meeting. La Spezia, Italy. 
Kent, Sherman. 1964. "Words of Estimative Probability." Journal of the American Intelligence Professional 8 (4): 49-65. Center for the Study of Intelligence.

Lahneman, William J. 2010. “The Need for a New Intelligence Paradigm.” International Journal of Intelligence and CounterIntelligence 23 (2): 201-25. doi:10.1080/08850600903565589.

Leung, Wanda, Bram Noble, Jill Gunn, and Jochen A. G. Jaeger. 2015. "A Review of Uncertainty Research in Impact Assessment." Environmental Impact Assessment Review 50: 116-23. doi:10.1016/j.eiar.2014.09.005.

Lipshitz, Raanan, and Orna Strauss. 1997. "Coping with Uncertainty: A Naturalistic Decision-Making Analysis.” Organizational Behavior and Human Decision Processes 69 (2): 149-63. doi:10.1006/obhd.1997.2679.

Lofstedt, Ragnar, Maeve McLoughlin, and Magda Osman. 2017. "Uncertainty Analysis: Results from an Empirical Pilot Study. A Research Note." Journal of Risk Research : 111. doi:10.1080/13669877.2017.1313768.

Lynham, S. A. 2002. "The General Method of Theory-Building Research in Applied Disciplines." Advances in Developing Human Resources 4 (3): 221-41. doi:10.1177/1523422302043002.

Mandel, David R. 2009. "Applied Behavioural Science in Support of Intelligence: Experiences in Building a Canadian Capability (Commissioned Report)." In Workshop of the Committee on Field Evaluation of Behavioral and Cognitive Sciences-Based Methods and Tools for Intelligence and Counterintelligence for the Division of Behavioral and Social Sciences and Education (DBASSE), 20. Toronto, Canada. http://citeseerx.ist.psu.edu/viewdoc/download?doi=10.1.1.187.4622\&rep=rep1\&type=pd f.

Mandel, David R., Alan Barnes, and Karen Richards. 2014. "A Quantitative Assessment of the Quality of Strategic Intelligence Forecasts.” Technical Report DRDC, TorontoTR 2013-036.

Mandel, David R., and Alan Barnes. 2014. "Accuracy of Forecasts in Strategic Intelligence." Proceedings of the National Academy of Sciences of the United States of America 111 (30): 10984-89. doi:10.1073/pnas.1406138111.

Marchio, Jim. 2014. "Analytic Tradecraft and the Intelligence Community: Enduring Value, Intermittent Emphasis.” Intelligence and National Security 29 (2): 159-83. doi:10.1080/02684527.2012.746415. 
Marrin, Stephen. 2004. "Preventing Intelligence Failures by Learning from the Past." International Journal of Intelligence and CounterIntelligence 17 (4): 655-72. doi:10.1080/08850600490496452.

Mastrandrea, Michael D., Christopher B. Field, Thomas F. Stocker, Ottmar Edenhofer, Kristie L. Ebi, David J. Frame, Hermann Held, et al. 2010. "Guidance Note for Lead Authors of the IPCC Fifth Assessment Report on Consistent Treatment of Uncertainties." Intergovernmental Panel on Climate Change. http://pubman.mpdl.mpg.de/pubman/item/escidoc:2147184/component/escidoc:2147185 /uncertainty-guidance-note.pdf.

Ministry of Defence UK. 2014. "Strategic Trends Programme- Future Operating Environment 2035 (First Edition)."

https://www.gov.uk/government/uploads/system/uploads/attachment_data/file/646821/2 0151203-FOE_35_final_v29_web.pdf.

Moore, David T. 2011. Sensemaking: A Structure for an Intelligence Revolution. Edited by C.Q. Thurston. Washington, D.C.: National Defense Intelligence College. http://www.au.af.mil/au/awc/awcgate/dia/ndic_moore_sensemaking.pdf.

National Research Council. 2010. Field Evaluation in the Intelligence and Counterintelligence Context: Workshop Summary. Washington, D.C.: National Academies Press.

NATO. 2010. “AJP-01 ( D )- Allied Joint Doctrine.” In Allied Joint Publication, 1:130. NATO Standardization Agency.

NATO. 2011. "AJP-3 (B)- Allied Joint Doctrine for the Conduct of Operations." In Allied Joint Publication, 3:120. NATO Standardization Agency.

NATO. 2016. “AJP-2.1; Edition B, Version 1, Allied Joint Doctrine for Intelligence Procedures." In Allied Joint Publication, Edition B,:92. NATO Standardization Office.

NATO. 2018. "AJP-3 ( C ), Edition C, Version 1, Allied Joint Doctrine for the Conduct of Operations." In Allied Joint Doctrine, Ratificati, 162. NATO Standardization Office.

NATO/ ACT. 2011. "Bi-Strategic Command; Knowledge Development." In Pre-Doctrinal Handbook, 45. NATO.

NATO RTO. 2012. "NATO Guide for Judgement-Based Operational Analysis in Defence Decision Making: Code of Best Practice for 'Soft' Operational Analysis.” RTO Technical Report, TR-SAS-087, Brussels. https://www.sto.nato.int/publications/STO Technical Reports/RTO-TR-SAS-087/\$TR-SAS-087-ALL.pdf. 
ODNI. 2015. "Intelligence Community Directive (ICD) 203- Analytic Standards." Washington, D.C.: Office of the Director of National Intelligence.

http://www.dni.gov/index.php/intelligence-community/ic-policies-reports/intelligencecommunity-directives.

Odom, William E. 2008. "Intelligence Analysis." Intelligence and National Security 23 (3): 316-32. doi:10.1080/02684520802121216.

Peterson, Joshua J. 2008. "Appropriate Factors to Consider When Assessing Analytic Confidence in Intelligence Analysis,” MSc thesis, Mercyhurst College, PA. http://c.ymcdn.com/sites/www.scip.org/resource/resmgr/White_Papers/PetersonAppropriate-Factors.pdf.

SHAPE. 2013. "Allied Command Operations Comprehensive Operations Planning Directive, Interim v 2.0.” In NATO Planning Directive, 444. Belgium: NATO/ Supreme Headquarters Allied Powers Europe.

Tansey, Oisín. 2007. "Process Tracing and Elite Interviewing: A Case for Non-Probability Sampling.” PS: Political Science and Politics 40 (4): 765-72. doi:10.1017/Si049096507071211.

Tversky, A., and D. Kahneman. 1974. "Judgment under Uncertainty: Heuristics and Biases." Science 185 (4157): 1124-31. doi:10.1126/science.185.4157.1124.

UK MOD. 2011. "Understanding and Intelligence Support to Joint Operations (JDP 2-00)." In Joint Doctrine Publication, 3rd ed., 155. DCDC.

US Government. 2009. “A Tradecraft Primer : Structured Analytic Techniques for Improving Intelligence Analysis Prepared by the US Government March 2009." A Primer of Analytic Techniques. https://www.cia.gov/library/center-for-the-study-ofintelligence/csi-publications/books-and-monographs/Tradecraft Primer-apr09.pdf.

US Joint Chiefs of Staff. 2009. "Joint Publication 2-01.3; Joint Intelligence Preparation of the Operational Environment.” In Joint Publication, 285. US CJCS.

US Joint Chiefs of Staff. 2013. “Joint Publication 2-0 Joint Intelligence.” In Joint Publications, 144. US CJCS.

Vrist Rønn, Kira, and Simon Høffding. 2013. “The Epistemic Status of Intelligence: An Epistemological Contribution to the Understanding of Intelligence." Intelligence and National Security 28 (5): 694-716. doi:10.1080/02684527.2012.701438. 
Walker, W.E., P. Harremoës, J. Rotmans, J.P. van der Sluijs, M. B. A. van Asselt, P. Janssen, and M.P. Krayer von Krauss. 2003. "Defining Uncertainty: A Conceptual Basis for Uncertainty Management in Model-Based Decision Support." Integrated Assessment 4 (1): 5-17. doi:10.1076/iaij.4.1.5.16466.

Walker, Warren E., Marjolijn Haasnoot, and Jan H. Kwakkel. 2013. "Adapt or Perish: A Review of Planning Approaches for Adaptation under Deep Uncertainty." Sustainability (Switzerland) 5 (3): 955-79. doi:10.3390/su5030955.

Walker, Warren E., Robert J. Lempert, and Jan H. Kwakkel. 2013. "Deep Uncertainty.” In Encyclopedia of Operations Research and Management Science, 395-402. Boston, MA: Springer US. doi:10.1007/978-1-4419-1153-7_1140.

Weiss, Charles. 2007. "Communicating Uncertainty in Intelligence and Other Professions." International Journal of Intelligence and CounterIntelligence 21 (1): 57-85. doi:10.1080/08850600701649312. 